\title{
Audiometría de Estado Estable
}

\section{Steady state audiometry}

Pablo Cabello $\mathrm{E}^{1}$, Jorge Caro $\mathrm{L}^{2}$.

\begin{abstract}
RESUMEN
El uso de pruebas electrofisiológicas ha sido una útil herramienta en la evaluación auditiva de pacientes con hipoacusia no susceptibles de evaluación con pruebas audiológicas conductuales.

La Audiometría de Estado Estable (AEE) es una prueba desarrollada recientemente que mediante la estimulación con tonos modulados en frecuencia y amplitud evoca una respuesta detectada en el electroencefalograma mediante técnicas de procesamiento computacional.

Esta técnica permite una estimación frecuencia específica del audiograma lo que es de especial utilidad en pacientes candidatos al uso de implantes cocleares.

Los métodos actuales muestran una buena correlación con el audiograma conductual en pacientes con hipoacusia severa, pero con mayor diferencia de umbral en pacientes con audición normal.

La AEE se ha constituido como una útil herramienta para complementar la batería de pruebas audiológicas usadas actualmente, sin embargo aún es necesario establecer nuevos protocolos que permitan mejorar la exactitud del examen.
\end{abstract}

Palabras clave: Audiometría de Estado Estable, evaluación auditiva, test electrofisiológico, hipoacusia sensorioneural.

\section{ABSTRACT}

The use of electrophysiological tests has been a useful tool in the auditory evaluation of hypoacusia patients non-susceptible to evaluation with behavioral audiological tests.

Steady state audiometry (SSA) is a recently developed test that, by means of stimulation with amplitude and frequency modulated tones, evokes an electroencephalogram response detected by computer processing techniques.

This technique allows for a frequency-specific evaluation of the audiogram, which is especially useful in patients that are being considered for cochlear implant.

Current methods show a good correlation with the behavioral audiogram in patients with severe hypoacusia, but with a higher threshold difference in patients with normal audition.

SSA constitutes a useful tool to complement the audiological test batteries currently used. However, new protocols with improved test accuracy are still needed.

Key words. Steady state audiometry, auditive evaluation, electrophysiological test, sensorineural hypoacusia.

1 Médico-Cirujano. Hospital Clínico Pontificia Universidad Católica de Chile.

2 Otorrinolaringólogo. Departamento de Otorrinolaringología. Hospital Clínico Pontificia Universidad Católica de Chile. 


\section{INTRODUCCIÓN}

La audiometría convencional es la prueba de elección para la evaluación del umbral auditivo en pacientes con hipoacusia. Sin embargo esta prueba posee limitaciones en cuanto exige la cooperación del paciente para informar si percibe o no los tonos o la voz hablada. La audiometría formal esta indicada en niños mayores a 4 años ${ }^{1}$ y adultos que sean capaces de contestar a las distintas intensidades de sonido. Para la evaluación de pacientes menores a 4 años se han desarrollado variantes a la audiometría formal que evalúan, mediante juegos o condicionamiento, cambios conductuales efectuados por el sujeto ante la percepción de sonidos 0 voz hablada ${ }^{1,2}$. Sin embargo, estas pruebas presentan igualmente limitaciones en la evaluación de pacientes que no presentan cambios conductuales evaluables como los recién nacidos ${ }^{3}$ y niños 0 adultos no cooperadores.

El advenimiento de las pruebas electrofisiológicas ha permitido evaluar a aquellas personas que no son candidatas a pruebas conductuales 0 en los cuales aparecen dudas en cuanto a la confiabilidad de los registros audiométricos. Dentro de las pruebas electrofisiológicas más usadas se encuentran las emisiones otoacústicas y los potenciales auditivos evocados de tronco cerebral o BERA (Brainstem Evoked Response Audiometry).

El BERA consiste en la estimulación auditiva mediante la emisión de clicks (sonidos breves que incluyen un espectro amplio de frecuencia para lograr una mayor estimulación de la vía auditiva) a diferentes intensidades, registrándose la respuesta mediante el uso de electrodos colocados en la región frontal y mastoides del paciente. Esta prueba permite estimar la sensibilidad y la integridad de la vía auditiva. Sin embargo, no provee información para cada frecuencia ${ }^{4}$, por lo que no permite una estimación del audiograma.

En 1980, Galambos y cols ${ }^{5}$ describen la detección mediante técnicas de sustracción computacional desde las señales captadas por el electroencefalograma, de un potencial auditivo evocado graficado como 3 ó 4 ondas a una frecuencia aproximada de $40 \mathrm{~Hz}$ y que responde en forma de una onda estable a estímulos realizados a una frecuencia de $40 \mathrm{~Hz}$. Además esta respuesta apareció a umbrales similares a los detectados por el audiograma para las distintas frecuencias. Surgía así la base para la Audiometría de Estado Estable (AEE).

\section{BASES FISIOLÓGICAS}

La respuesta auditiva de estado estable (RAEE) estaría construida a partir de la descarga de generadores troncoencefálicos y corticales bilaterales, estos últimos en el plano supratemporal. Estos generadores responderían en forma diferenciada a las distintas modulaciones de frecuencias de estimulación, con predominancia del generador troncoencefálico a modulaciones alrededor de los $80 \mathrm{~Hz}$ y de la corteza a modulaciones alrededor de los $40 \mathrm{~Hz}^{6}$. La fase de la RAEE varía junto con la frecuencia del estímulo, fenómeno conocido como fijación de fase (phase-locking). Modelos experimentales sugieren que aproximadamente $25 \%$ de las células de la corteza auditiva originarían la fijación de fase, las cuales responden a los cambios de la frecuencia de estimulación?

Existen dos teorías sobre la forma de generación de la RAEE. La primera sugiere la sobreposición de potenciales llamados respuesta de latencia media (RLM) los cuales aparecen 8 a 80 milisegundos después de efectuado el estímulo y se manifiestan como ondas a intervalos de 25 milisegundos, que se sobreponen al estimular a una frecuencia de $40 \mathrm{~Hz}$, generando una respuesta estable de $40 \mathrm{~Hz}^{5,6}$. La segunda teoría propone la existencia de unidades neuronales con un ritmo de descarga espontáneo el cual presentaría una mayor resonancia con estímulos de $40 \mathrm{~Hz}^{6}$.

La RAEE con modulaciones mayores a $60 \mathrm{~Hz}$ persiste a pesar del uso de sedación 0 analgesia ${ }^{4}$, pero esto no ocurre con modulaciones de $40 \mathrm{~Hz}$, donde el uso de tiopental o propofol la atenúan8.

\section{CARACTERÍSTICAS DEL PROCEDIMIENTO}

En la realización de una AEE se utilizan audífonos insertables, electrodos de superficie y un computador para procesar la señal. Los audífonos son insertados en el canal auricular del oído seleccionado, emitiendo estímulos en forma de tonos frecuencia-específicos los cuales son modulados en amplitud (AM) y frecuencia (FM). 
Los tonos estimulan el aparato coclear y el sistema nervioso central (SNC), cuya respuesta es detectada mediante electrodos de superficie usualmente ubicados en las posiciones $\mathrm{Cz}, \mathrm{Iz}$, mastoides y Fpz (tierra) (Figura 1). La derivación Cz-lz presenta una menor cantidad de «ruido» con señales de $90 \mathrm{~Hz}$ de $\mathrm{AM}^{9}$. La señal captada es enviada a un computador, el cual mediante análisis estadísticos determina si la señal corresponde sólo a «ruido» o corresponde a una respuesta auditiva de estado estable.

La AEE permite la determinación de umbrales auditivos en frecuencias que van entre los $250 \mathrm{y}$ los $8.000 \mathrm{~Hz}$ con estímulos sobre 127,8 dB de pérdida auditiva ${ }^{10}$, a diferencia del BERA el cual presenta mayor similitud con el audiograma clásico en intensidades entre los 0 y $90 \mathrm{~dB}$ en niños ${ }^{11}$.

El BERA con burst de tonos usa estimulación auditiva con ondas sinusales entre 500 y $8 \mathrm{kHZ}$ aplicadas en trenes de 2, 40 más estímulos ${ }^{12}$. Este examen y la AEE son los que permiten una estimación frecuencia específica del audiograma, sin embargo el BERA con burst de tonos es más demoroso en su aplicación ya que la prueba para cada frecuencia, equivale a realizar un BERA con respuesta evocada por clicks $^{13}$. Esto presenta una desventaja especialmente en pacientes pediátricos en los cuales es más difícil mantener el reposo exigido por la prueba.

\section{AEE EN NIÑOS}

El advenimiento de los implantes cocleares ha sido un avance en la implementación de niños con hipoacusia severa o profunda que no presentan respuesta con audífono. El uso de estos implantes a edades tempranas muestra ventajas sobre su indicación en niños de edades más avanzadas ${ }^{14}$, siendo la indicación actual en niños mayores a 12 meses $^{15} \mathrm{e}$ incluso a edades menores en algunos estudios ${ }^{11}$.

La implementación cada vez más precoz ha hecho necesario el uso de técnicas objetivas que permitan la estimación del audiograma en este tipo de pacientes. El uso de BERA se limita a intensidades de 90 a $100 \mathrm{~dB}$ dependiendo de las características técnicas del equipo, limitando el estudio de hipoacusias más profundas. Además el espectro de frecuencias de estimulación con click es sobre los $1.000 \mathrm{~Hz}$, requiriendo el uso de burst de tonos para frecuencias menores ${ }^{11}$.

La AEE permite la evaluación de niños con hipoacusias severas a profundas, detectando aquellas frecuencias en las cuales hay remanentes de audición, aun cuando no son detectadas por el BERA $^{16}$, lo que es necesario para evaluar la elegibilidad para el implante coclear ${ }^{11}$. Este examen ha

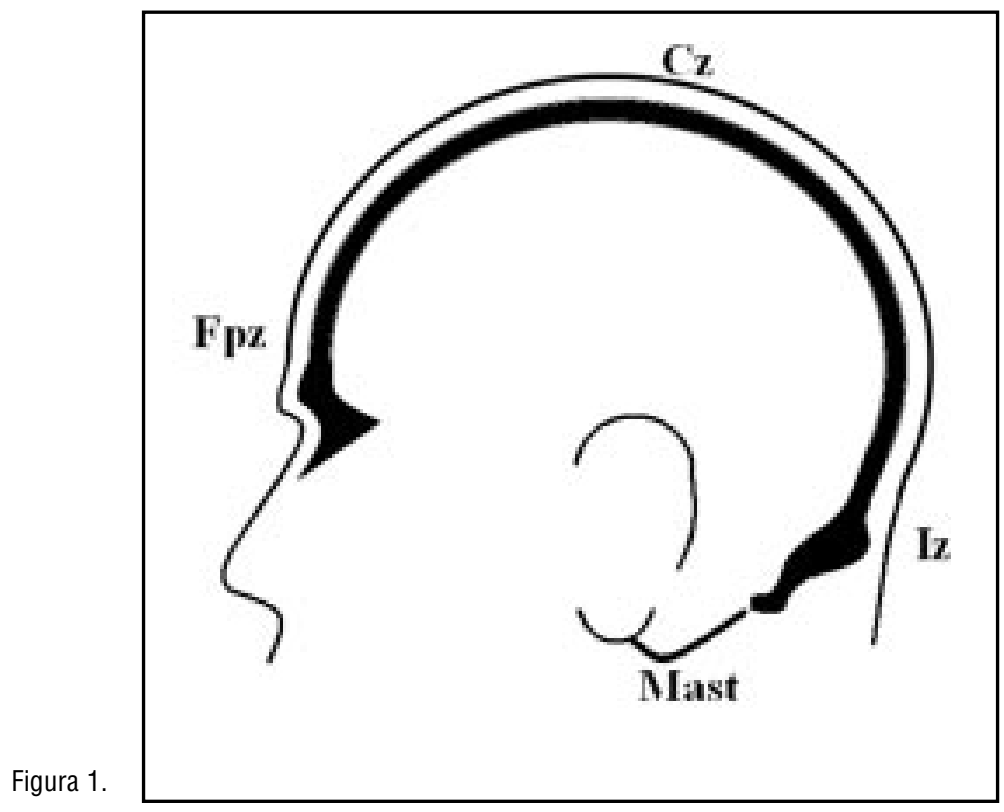


mostrado una buena correlación con el audiograma conductual, especialmente en pacientes con hipoacusia profunda ${ }^{4,17}$ con $69 \%$ de los umbrales ubicados entre $0 \mathrm{~dB}$ y $10 \mathrm{~dB}$ con respecto a los de la audiometría conductual, según Swanepoel y cols, y con resultados similares para otros estudios.

Sin embargo hay resultados variables para la capacidad de detección de una RAEE a frecuencias de $500 \mathrm{~Hz}$, presentando también a estas frecuencias las mayores diferencias con el audiograma conductual ${ }^{17,18}$, lo que podría deberse a una mayor variabilidad intrínseca debida a asincronía neuronal en las respuestas frente a estas frecuencias.

En el caso de la hipoacusia sensorioneural descendente, la AEE usando la técnica de phase-locking ha demostrado predecir la forma del audiograma entre los $500 \mathrm{~Hz}$ y $4.000 \mathrm{~Hz}$, sobreestimando el umbral en $15 \mathrm{a}$ $20 \mathrm{~dB}$ en las frecuencias mayores a $500 \mathrm{~Hz}^{10}$.

\section{AEE EN ADULTOS}

En adultos con audición normal existe una gran variabilidad en los resultados para determinar el umbral auditivo para la AEE a frecuencias de $500 \mathrm{y}$ $4.000 \mathrm{~Hz}$, existiendo una buena correlación entre los umbrales detectados por AEE y BERA ${ }^{19}$.

El umbral se ve afectado también por el nivel de conciencia del paciente, lográndose los menores umbrales con modulaciones alrededor de $40 \mathrm{~Hz}$ en vigilia y mayores a $70 \mathrm{~Hz}$ en pacientes dormidos ${ }^{19}$. Petitot y cols muestran que la modulación a $40 \mathrm{~Hz}$ produce umbrales más cercanos a la audiometría conductual que con modulaciones mayores a 70 $\mathrm{Hz}^{20}$ por lo que sugiere preferir la realización del examen a $40 \mathrm{~Hz}$ y en pacientes despiertos.

El umbral de la audiometría conductual presenta una diferencia relativamente elevada con el detectado por la AEE en pacientes con audición normal, a diferencia de lo que ocurre en la predicción en hipoacusia sensorioneural ${ }^{4}$.

La AEE ha demostrado ser confiable y reproducible al usar a los sujetos evaluados como sus propios controles, mostrando una buena correlación para frecuencias mayores a $1.000 \mathrm{~Hz}$, pero presentando nuevamente problemas en la determinación del umbral a frecuencias de $500 \mathrm{~Hz}^{21}$. Además se ha observado una alta sobreestimación del umbral auditivo a frecuencias de 8.000 y $12.000 \mathrm{~Hz}^{20}$.

\section{CONCLUSIONES}

La AEE se ha convertido en una útil herramienta para la evaluación de pacientes con hipoacusia, especialmente en pacientes en los que la evaluación con pruebas subjetivas es difícil. Sin embargo su uso debe complementarse con el resto de la batería de exámenes objetivos.

Dado la relativamente amplia diferencia entre el umbral establecido por la AEE y la audiometría conductual en pacientes con audición normal, no es posible utilizarla como examen médico-legal para diferenciar simulación de hipoacusia verdadera. Aún es necesario establecer nuevos protocolos y técnicas que permitan una mejor determinación del umbral auditivo en casos en que no se logran valores similares a los de la audiometría conductual, como por ejemplo en las frecuencias bajas y en ciertos tipos de audiograma.

\section{BIBLIOGRAFÍA}

1. Cunningham M, Cox E, Comitee on Practice and Ambulatory Medicine and the Section on OtolaRYNGOLOGT AND BRONCHOESOPHAGOLOGY. Hearing Assessment in Infants and Children: Recommendations Beyond Neonatal Screening. Pediatrics 2003; 111(2): 436-40.

2. SIRIMANKA KS. Management of the hearing impaired infant. Semin Neonatol 2001; 6: 51119.

3. Hicks CB, Tharpe AM, Ashmead DH. Behavioral auditory assessment of young infants: methodological limitations or natural lack of auditory responsiveness? Am J Audiol 2000; 9(2): 124-30.

4. Canale A, lacilla M, Cavalot al, Albera R. Auditory steady-state responses and clinical applications. Eur Arch Otorhinolaryngol 2006; 263: 499-503.

5. Galambos R, Makeig S, Talmachoff PJ. A 40-Hz auditory potential recorded from the human scalp. Proc Natl Acad Sci USA 1981; 78(4): 2643-47.

6. Herdman at, Lins 0, Van Roon P, Stapells DR, Scherg M, PInton TW. Intracerebral Sources of Human Auditory Steady-State Responses. Brain Topogr 2002; 15(2): 69-86. 
7. Swanepoel D, Hugo R. Estimations of auditory sensitivity for young cochlear implant candidates using the ASSR: preliminary results. Int J Audiol 2004; 43(7): 377-82.

8. Plourde G. Auditory evoked potentials. Best Pract Res Clin Anaesthesiol 2006; 20(1): 12939.

9. Van Der Reijden CS, Mens lH, Snik aF. Comparing signal to noise ratios of amplitude modulation following responses from four EEG derivations in awake normally hearing adults. Audiology 2001; 40(4): 202-7.

10. Ballay C, Tonini NR, Waninger T, Yoon C, Manolidis S. Steady-State Response Audiometry in a group of patients with steeply sloping sensorineural hearing loss. Laryngoscope 2005; 115(7): 1243-46.

11. Roberson JB JR, O'Rourke C, Stidham KR. Auditory steady-state response testing in children: Evaluation of a new technology. Otolaryngol Head Neck Surg 2003; 129(1): 107-13.

12. De Sebastián G. Audiología práctica 5ae edición. Buenos Aires: Editorial Médica Panamericana 1999: 234.

13. LUTS H, Wouters J. Comparison of MASTER and AUDERA for measurement of auditory steadystate responses. Int J Audiol 2005; 44(4): 244-53.

14. Anderson I, Weichbold V, D'Haese PS et al. Cochlear implantation in children under the age of two-what do the outcomes show us? Int $J$ Pediatr Otorhinolaryngol 2004; 68(4): 425-31.

15. Gregg RB, Wiorek LS, Arvedson JC. Pediatric Audiology: A Review. Pediatr Rev 2004; 25(7): 224-34.

16. Patel ad, Balaban E. Human Auditory Cortical Dynamics During Perception of Long Acoustic Sequences: Phase Tracking of Carrier Frequency by the Auditory Steady-state Response. Cereb Cortex 2004 Jan; 14(1): 35-46.

17. Swanepoel D, Hugo R, Roode R. Auditory steadystate responses for children with severe to profound hearing loss. Arch Otolaryngol Head Neck Surg 2004; 130(5): 531-35.

18. Lins OG, Picton TW, Boucher BL et aL. Frequency-specific audiometry using Steadystate Responses. Ear Hear 1996; 17(2): 81-9.

19. Cone-Wesson B, Dowell RC, Tomlin D, Rance G, Ming WJ. The Auditory Steady-state Response: Comparisons with the Auditory Brainstem Response. J Am Acad Audiol 2002; 13(4): 173-87.

20. Petitot C, Collet L, Durrant JD. Auditory steady-state responses (ASSR): effects of modulation and carrier frequencies. Int $J$ Audiol 2005; 44(10): 567-73.

21. Kaf Wa, Sabo DL, Durrant JD, Rubinstein E. Reliability of electric response audiometry using $80 \mathrm{~Hz}$ auditory steady-state responses. Int J Audiol 2006; 45(8): 477-86. 